\title{
ÍNDICE ANALÍTICO
}

Acceso público a los documentos del Tribunal de Cuentas Europeo, UE-1427 Acta relativa a las condiciones de adhesión de la República de Bulgaria y de Rumanía y a las adaptaciones de los Tratados en los que se fundamenta la Unión Europea, UE-1381

Actualización de la relación de los aeropuertos comunitarios, UE-1344

Acuerdo de cooperación entre la Comunidad Europea de la Energía Atómica y el Gobierno de la República de Kazajstán en el ámbito de la fusión termonuclear controlada, UE-1372, UE-1384

Acuerdo de cooperación entre la Comunidad Europea y el Principado de Andorra, UE-1368

Acuerdo entre la Comunidad Económica Europea y la República de Costa de Marfil sobre la pesca, UE-1335

Acuerdo entre la Comunidad Económica Europea y la República Democrática de Madagascar sobre la pesca, UE-1334, UE-1364

Acuerdo entre la Comunidad Europea y el Principado de Andorra en materia de fiscalidad de los rendimientos del ahorro en forma de pago de intereses, UE-1355, UE-1367

Acuerdo entre la Comunidad Europea y el Principado de Liechtenstein en materia de fiscalidad de los rendimientos del ahorro en forma de pago de intereses, UE-1354, UE-1366

Acuerdo entre la Comunidad Europea y el Principado de Mónaco en materia de fiscalidad de los rendimientos del ahorro en forma de pago de intereses, UE-1353, UE-1365

Acuerdo entre la Comunidad Europea y la República de San Marino en materia de fiscalidad de los rendimientos del ahorro en forma de pago de intereses, UE-1356, UE-1369

Acuerdo entre la Comunidad Europea y la República de Serbia sobre el comercio de productos textiles, UE-1363

Acuerdo entre la Comunidad Europea y los Estados Unidos de América sobre medidas sanitarias para proteger la salud pública y la sanidad animal en el comercio de animales vivos y de productos de origen animal, UE-1371

Acuerdo entre la Unión Europea y la Antigua República Yugoslava de Macedonia sobre procedimientos de seguridad para el intercambio de información clasificada, UE-1428

Acuerdo entre la Unión Europea y la República Argentina sobre la participación de la República Argentina en la operación militar de gestión de crisis de la Unión Europea en Bosnia y Herzegovina (Operación Althea), UE-1432 
Acuerdo entre la Unión Europea y la República de Bulgaria sobre procedimientos de seguridad para el intercambio de información clasificada, UE-1430 Acuerdo entre la Unión Europea y Nueva Zelanda sobre la participación de Nueva Zelanda en la Operación Militar de gestión de crisis de la Unión Europea en Bosnia y Herzegovina (Operación Althea), UE-1431

Acuerdo entre la Unión Europea y Rumanía sobre procedimientos de seguridad para el intercambio de información clasificada, UE-1429

Acuerdo Europeo por el que se crea una asociación entre las Comunidades Europeas y sus Estados miembros, por una parte, y la República de Bulgaria, por otra, para tener en cuenta la adhesión a la Unión Europea de la República Checa, la República de Estonia, la República de Chipre, la República de Hungría, la República de Letonia, la República de Lituania, la República de Malta, la República de Polonia, la República de Eslovenia y la República Eslovaca, UE-1373

Acuerdo Europeo por el que se crea una asociación entre las Comunidades Europeas y sus Estados miembros, por una parte, y Rumanía, por otra, para tener en cuenta la adhesión a la Unión Europea de la República Checa, la República de Estonia, la República de Chipre, la República de Hungría, la República de Letonia, la República de Lituania, la República de Malta, la República de Polonia, la República de Eslovenia y la República Eslovaca, UE-1374

Acuerdo por el que se establece una asociación entre la Comunidad Europea y sus Estados miembros, por una parte y la República de Chile, por otra, UE-1362

Adhesión de la República Checa, la República de Estonia, la República de Chipre, la República de Letonia, la República de Lituania, la República de Hungría, la República de Malta, la República de Polonia, la República de Eslovenia y la República Eslovaca al Convenio relativo a la supresión de la doble imposición en caso de corrección de beneficios de empresas asociadas, UE-1357

Aditivo en la alimentación animal, UE-1314

Aditivos ya permitidos en la alimentación animal, UE-1313

Administración General del Estado: EST-24, EST-25

Agencia Comunitaria de Control de la Pesca, UE-1336

Agencia Europea para la gestión de la cooperación operativa en las fronteras exteriores de los Estados miembros de la Unión Europea, UE-1303, UE-1455

Agricultura: CAT- 1; AND-1; AND-2; AND-3

Altos Cargos: CAT-7; CAT-8

Animales: EXT- 1

Aplicación de la asistencia comunitaria, UE-1302 
Aplicación de los regímenes de seguridad social a los trabajadores por cuenta ajena, los trabajadores por cuenta propia y los miembros de sus familias que se desplazan dentro de la Comunidad, UE-1340

Arrendamientos rústicos: GAL-1

Asistencia jurídica: VAL-2

Autorización permanente de aditivos en la alimentación animal, UE-1324, UE-1326

Bienes de las Entidades Locales: AND-8

Buenas prácticas clínicas respecto a los medicamentos en investigación de uso humano, UE-1389

Capital europea de la cultura para los años 2005 a 2019, UE-1421

Capital europea de la cultura para los años 2005 a 2019, UE-1421

Catastro Inmobiliario: EST-28

Caza y Pesca: NAV-2; RIO-1

Código aduanero comunitario, UE-1308, UE-1309

Colegios Profesionales: CMN-1

Comunicación audiovisual: CAT-4

Comunicaciones electrónicas de banda ancha a través de la red eléctrica, UE-1405

Comunicaciones entre los Estados miembros y la Comisión en el sector de la leche y de los productos lácteos, UE-1315

Comunidad Valenciana: EST-23

Confederaciones Hidrográficas: AND-4

Conferencia de las Partes del Año 2005 encargada del examen del Tratado sobre la no proliferación de las armas nucleares, UE-1447

Consejo de Asociación UE-Marruecos normas necesarias para la aplicación de las normas de competencia, UE-1382

Consejo de Estabilización y Asociación UE-Croacia, UE-1370

Consumidores y usuarios: DRC-1; CMN-4

Convenio de la Organización Internacional del Trabajo sobre los documentos de identidad de la gente de mar (Convenio $\left.\mathrm{n}^{\circ} 185\right)$, UE-1339

Convenio sobre el acceso a la información, la participación del público en la toma de decisiones y el acceso a la justicia en materia de medio ambiente, UE-1406

Cooperación de la Comunidad con la Organización de las Naciones Unidas para la Agricultura y la Alimentación (FAO), UE-1332

Decisión de la Comisión sobre las solicitudes de adhesión a la Unión Europea presentadas por la República de Bulgaria y por Rumanía, UE-1375

Decisión del Consejo de la Unión Europea relativa a la admisión de la República de Bulgaria y Rumanía, UE-1378 
Declaración de conformidad y a los criterios de realización de los análisis de objetos de cerámica destinados a entrar en contacto con productos alimenticios, UE-1391

Depósitos y Consignaciones judiciales: EST-32

Discapacitados: AND-7

Disposiciones para la prevención, el control y la erradicación de determinadas encefalopatías espongiformes transmisibles, UE-1327

Educación: EST-22

Empleo: EST-20

Enajenación de participaciones públicas: EST-19

Entidades de Derecho Público: EST-26

Equipamientos comerciales: CAT-3

Escuelas Oficiales de Idiomas: AND-6

Espectáculos públicos: ARA-1

Estabilidad presupuestaria: EST-30, EST-31

Estadística: AND-9

Estadísticas sobre residuos, UE-1419

Estado Mayor de la Unión Europea, UE-1441

Estatuto de los ex-Presidentes: CAS-1

Etiqueta ecológica comunitaria, UE-1408, UE-1409, UE-1410, UE-1411, UE-1412, UE-1413, UE-1414

Eurojust: EST-33

Evaluación de las políticas públicas y de la calidad de los servicios públicos: NAV-3

Exigencias de información estadística del Banco Central Europeo, UE-1358, UE-1359

Explotaciones Ganaderas: AND-5

Familia: AND-10

Fecha límite para el compromiso de los fondos del noveno Fondo Europeo de Desarrollo (FED), UE-1402

Financiación de estudios, valoraciones de impacto y evaluaciones en los ámbitos de la seguridad alimentaria, la salud y el bienestar de los animales y la zootecnia, UE-1333

Fondo Europeo de Desarrollo, UE-1403

Fondo Europeo de Orientación y de Garantía Agrícola (FEOGA), UE-1317, UE-1318

Fondos de pensiones de empleo: EST-17

Formato para la transmisión de resultados de estadísticas sobre residuos, UE-1418 Formato técnico destinado a la producción de estadísticas comunitarias anuales del acero, UE-1417 
Franquicia: EST-16

Gastos de financiación de las intervenciones consistentes en compras, almacenamiento y comercialización, UE-1320

Gestión de activos exteriores de reserva del Banco Central Europeo por los bancos centrales nacionales, UE-1360

Grupo de expertos no gubernamentales en gobernanza empresarial y Derecho de sociedades, UE-1426

Grupo Europeo de Ética de la Ciencia y de las Nuevas Tecnologías, UE-1423

Homologación de los vehículos de motor de dos o tres ruedas, UE-1390

Horarios comerciales: CMN-2; CAT-2

Importaciones de determinados productos originarios de los Estados Unidos de América, UE-1307

Incendios forestales: CAS-2; CAS-3

Información y realización de pruebas a los importadores o fabricantes de determinadas sustancias prioritarias, UE-1385

Iniciativa Legislativa Popular: CAT-9

Iniciativa legislativa popular: EST-13

Inmigración: MAD-1

Inmovilizado no financiero de las Comunidades Europeas, UE-1301

Instituto cartográfico de Cataluña: CAT-5

Instituto Geológico de Cataluña: CAT-6

Instrumentos y procedimientos de la política monetaria del Eurosistema, UE-1361 IVA: EST-29

La educación y la formación en el marco del examen intermedio de la estrategia de Lisboa, UE-1420

La juventud en el marco de la evaluación intermedia de la estrategia de Lisboa, UE-1422

Límites presupuestarios de los pagos directos, UE-1316

Lista de terceros países cuyos nacionales están exentos de esa obligación en lo que respecta al mecanismo de reciprocidad, UE-1453

Lista de terceros países cuyos nacionales están sometidos a la obligación de visado para cruzar las fronteras exteriores, UE-1453

Listas de los procedimientos de insolvencia, los procedimientos de liquidación y los síndicos, UE-1341

Medida temporal de exclusión de la ayuda compensatoria para los productos comercializados procedentes de nuevos platanares, UE-1331

Medidas administrativas, fiscales y financieras: DRC-2; RIO-2; CAS-4; BAL-1; CAT-10; VAL-3; MAD-2

Medidas antidumping, UE-1349

Medidas definitivas de salvaguardia a las importaciones de salmón de piscifactoría, UE-1306 
Medidas en apoyo de la aplicación efectiva del mandato del Tribunal Penal Internacional para la ex Yugoslavia (TPIY), UE-1440

Medidas específicas de lucha contra el terrorismo, UE-1450

Medidas restrictivas adicionales contra Liberia, UE-1437

Medidas restrictivas contra Birmania/Myanmar, UE-1434

Medidas restrictivas contra Birmania/Myanmar, UE-1448

Medidas restrictivas contra Sudán, UE-1436, UE-1449

Medidas restrictivas en apoyo de la aplicación efectiva del mandato del Tribunal Penal Internacional para la ex Yugoslavia (TPI), UE-1433

Medidas restrictivas específicas dirigidas a determinadas personas y entidades con el fin de luchar contra el terrorismo, UE-1442

Medidas restrictivas específicas dirigidas contra determinadas personas y entidades asociadas con Usamah bin Ladin, la red Al-Qaida y los talibanes, UE-1435

Medidas restrictivas específicas dirigidas contra determinadas personas y entidades habida cuenta de la situación en Costa de Marfil, UE-1452

Medidas restrictivas respecto de la República Democrática del Congo, UE-1438, UE-1451

Medidas restrictivas respecto de Zimbabwe, UE-1439, UE-1443

Medio Ambiente: EST-37

Memoria histórica: AND-11

Menores: RIO-3; NAV-4

Mercado de valores: EST-18

Métodos de muestreo y de análisis para el control oficial del contenido de toxinas de Fusarium, UE-1395

Misión de asesoramiento y asistencia de la Unión Europea en materia de reforma del sector de la seguridad en la República Democrática del Congo (RDC), UE-1445

Modelos relativos al sistema de bases de datos relativos a los envases y residuos de envases, UE-1394

Modelos relativos al sistema de bases de datos, UE-1407

Montes: EST-38

Multas a una empresa por facilitar información incorrecta o engañosa, UE-1350

Municipios de gran población: VAL-1

Normas comunes de comercialización para determinados productos pesqueros, UE-1337

Normas comunes de seguridad aérea, UE-1345, UE-1346

Normas de desarrollo para controlar el cumplimiento de los objetivos de reutilización y valorización así como de reutilización y reciclado de los vehículos al final de su vida útil, UE-1398 
Normas particulares de ejecución de las disposiciones relativas a la asignación de una participación financiera de la Comunidad para la lucha fitosanitaria, UE-1386 Nuevas funciones para el Sistema de Información de Schengen, inclusive en materia de lucha contra el terrorismo, UE-1457

Nuevas sustancias psicotrópicas, UE-1456

Ordenación del territorio: AND-12

Ordenación vitivinícola: NAV-1

Organización común de mercados en el sector del lino y el cáñamo, UE-1325

Organización común del mercado vitivinícola, UE-1321

Patentes y marcas: EST-14

Patrimonio Histórico-Artístico: VAL-4; AND-13; AND-14; AND-15

Personal al servicio de las Administraciones Públicas: EST-27

Prácticas comerciales desleales de las empresas en sus relaciones con los consumidores en el mercado interior, UE-1397

Presupuestos Generales: DRC-3; RIO-4; NAV-5; CAS-5; BAL-2; GAL-2; CAT-11; EXT-2; VAL-5; CMN-5;MAD-4; PV-1

Prevención, gestión y resolución de conflictos en África, UE-1446

Procedimiento de consultas abierto con la República de Guinea en virtud del artículo 96 del Acuerdo de Cotonú, UE-1383

Procedimientos para las inspecciones de la Comisión en el ámbito de la protección marítima, UE-1347

Procedimientos penales: EST-34, EST-35

Procurador General: AST-1

Producción efectiva de aceite de oliva y el importe de la ayuda unitaria a la producción, UE-1328

Productos cosméticos, UE-1393

Productos fitosanitarios que contienen microorganismos, UE-1388

Programa comunitario plurianual para el fomento de un uso más seguro de Internet y las nuevas tecnologías en línea, UE-1416

Programa de control específico relativo a la recuperación de poblaciones de bacalao, UE-1338

Propiedad intelectual e industrial: EST-36

Protección y fomento del arbolado urbano: MAD-3

Protocolo relativo a las condiciones y al procedimiento de admisión de la República de Bulgaria y de Rumanía a la Unión Europea, UE-1380

Publicidad: AND-16

Radio y televisión: EST-39

Red segura de información y coordinación para los servicios de gestión de la migración de los Estados miembros, UE-1454

Régimen común aplicable a las importaciones de algunos productos textiles originarios de países terceros, UE-1310, UE-1311 
Régimen de ayudas concedido por España a las industrias de aceite de orujo de aceituna, UE-1351

Régimen de ayudas ejecutado por el Reino de España en favor de la compañía aérea Intermediación Aérea SL, UE-1352

Régimen lingüístico de la Comunidad Económica Europea, UE-1304

Régimen lingüístico de la Comunidad Europea de la Energía Atómica, UE-1304

Regímenes de ayuda directa en el marco de la política agrícola común, UE-1319

Regímenes de ayuda, UE-1323

Registro de Denominaciones de Origen Protegidas y de Indicaciones Geográficas Protegidas (Miel de Granada), UE-1424

Reglamento interno del Consejo de Ministros ACP-CE, UE-1300

Relación estadística del transporte marítimo de mercancías y pasajeros, UE-1348

Representante Especial de la Unión Europea para el Cáucaso Meridional, UE-1444

Reproducción humana asistida: EST-40

Requisitos de seguridad que deben establecer las normas europeas en relación con los artículos de ocio flotantes para usar en o sobre el agua, UE-1399

Residuos de aparatos eléctricos y electrónicos, UE-1415

Residuos de plaguicidas sobre y en los cereales y en determinados productos de origen vegetal, UE-1330, UE-1392

Resolución legislativa del Parlamento Europeo sobre la solicitud de ingreso de la República de Bulgaria, UE-1376

Resolución legislativa del Parlamento Europeo sobre la solicitud de ingreso de Rumanía, UE-1377

Respeto de los derechos de propiedad intelectual, UE-1425

Sector Eléctrico: CAN-1

Sector energético: EST-21

Seguro de responsabilidad civil derivada de la circulación de vehículos automóviles, UE-1342, UE-1396

Sistema de identificación especial para animales de la especie bovina mantenidos por razones culturales e históricas, UE-1322

Sistema de preferencias arancelarias generalizadas, UE-1312

Sociedad de la información: AND-17

Sociedades Anónimas: EST-15

Suministro de líneas arrendadas en la Unión Europea, UE-1404

Sustancias activas etoxazol y tepraloxidim, UE-1329

Televisión local: AND-18; AND-19

Toxinas de Fusarium, UE-1387, UE-1395 
Transporte por carretera: $\mathbf{C M N - 6}$

Transposición al Derecho nacional de las Directivas que afectan al mercado interior, UE-1401

Tratado entre el Reino de Bélgica, la República Checa, el Reino de Dinamarca, la República Federal de Alemania, la República de Estonia, la República Helénica, el Reino de España, la República Francesa, Irlanda, la República Italiana, la República de Chipre, la República de Letonia, la República de Lituania, el Gran Ducado de Luxemburgo, la República de Hungría, la República de Malta, el Reino de los Países Bajos, la República de Austria, la República de Polonia, la República Portuguesa, la República de Eslovenia, la República Eslovaca, la República de Finlandia, el Reino de Suecia y el Reino Unido de Gran Bretaña e Irlanda del Norte (Estados miembros de la Unión Europea) y la República de Bulgaria y Rumanía, relativo a la adhesión de la República de Bulgaria y de Rumanía a la Unión Europea, UE-1379 Tributos: NAV-6; VAL-6; EXT-3; EXT-4; CMN-7; NAV-7; CMN-8

Tropa y marinería: EST-12

Turismo: CMN-9; AND-20; AND-21

Urbanismo: VAL-7; CMN-10

Uso oficial de otras lenguas en el Consejo y, en su caso, en otras instituciones y órganos de la Unión Europea, UE-1305

Utilización de las tierras retiradas de la producción con vistas a la obtención de materias primas, UE-1323

Víctimas del terrorismo: EXT-5

Violencia de género: MAD-5 